symptoms of distress (respiration 95 per min., pulse 120 per min.) and was slightly blown; the heifers, although full in the flank, were neither blown nor showing signs of discomfort. Blood samples taken at this stage were analysed for hydrocyanic acid content; the bullock's blood contained 0.1 mgm. hydrocyanic acid per $100 \mathrm{ml}$. blood, while the heifers' had $0.04 \mathrm{mgm}$. per $100 \mathrm{ml}$. blood.

The possible role of hydrocyanic acid in the etiology of acute bloat in sheep has been considered before by Clark and Quin?, but they prefer the 'saponin foam theory' of its pathogenesis (see also (lark $\left.{ }^{8}\right)$.

It seems probable to us that in a complex syndrome like bloat several factors may operate; a satisfactory theory of the cause must not only account for the sequence of symptoms in the animal, but must also explain observations of agriculturists on pre. disposing environmental conditions. We believe that our findings are significant in this connexion.

We wish to acknowledge our grateful thanks to the Welsh Plant Breeding Station for providing facilities in the field.

' Ferguson, W. S., Nature, 161, 816 (1948). 'Cole, H. H., Huffman, C. F., Kleiber, Max, Olson, T. M., and Schalk,
A. F., J. Anim. Sci., 4, 183 (1945).

'Van der Walt, S. J., Onderstepoort J., 19, 79 (1944).

'Melville, J., and Doak, B. W., N.Z. J. Sci. and Tech., 22 B, 67 B (1944).

'Dougherty, R. W., J. Amer. Vet. Med. Assoc., 99, 110 (1941).

- Hindmarsh, W. L., J. Council of Sci. Indust. Res., 3, 112 (1930).

'Clark, R., and Quin, J. L., Onderstepoort J., 20, 209 (1945).

- Clark, R., Onderstepoort J., 28, 189 (1948).

\section{DETERMINATION OF ABSOLUTE INTENSITIES OF X-RAY REFLEXIONS FROM RELATIVE} INTENSITY DATA

\author{
Prof. S. H. YÜ \\ Departmeny of Physics, National Tsing-hua University,
}

$\mathrm{B}$ ECENTLY, owing to the advent of various ngles of Bragg reflexions from a crystal, a convenient method of determination of absolute intensities of Bragg reflexions seems urgently needed ${ }^{2}$. In 1942 I proposed a simple method of determination of absolute from relative intensities ${ }^{3}$, which has since been made rigorous and so should be reliable in general cases if used properly. Accordingly, I present here the final result of the method, which is applicable to practical cases immediately.

Suppose we have a crystal containing $t$ atoms in the unit cell, the number of shell electrons of the $i$ th atom being $Z_{i}$. Let the relative intensities of reflexions for the crystal at room temperature be denoted by $I^{\prime}(h k l)$ for $h k l$ and $I^{\prime}(000)$ for 000 . We have

$$
|F(h k l)|^{2}=\frac{F^{2}(000)}{I^{\prime}(000)} I^{\prime}(h k l) .
$$

We see that if we can determine $I^{\prime}(000)$ we can deduce $\left|F^{\prime}(h k l)\right|^{2}$ from $I^{\prime}(h k l)$, for we have the known $F(000)=\sum_{i=1}^{t} Z_{i}$. Let us introduce

$$
\bar{f}(h k l)=\sum_{i=1}^{t} f_{i}(h k l) / \sum_{i=1}^{t} Z_{i},
$$

where $f_{i}(h k l)$ is the atomic structure factor at room temperature of the ith atom in the cell. If only $f_{i}(h k l)$ for atoms at rest are available, such as those obtained from James and Brindley's table, suitable temperature correction must be made in order to obtain the room temperature value of $f_{i}(h k l)$. We further form the sum $\sum_{i=1}^{t} Z_{i}{ }^{2}$. Then from the theory of a new X-ray synthesis developed by me, it can be shown that:

$$
I^{\prime}(000)=F^{2}(000) \frac{\sum^{\prime} U_{n_{1} h} U_{n_{2} k} U_{n_{3}} I^{\prime}(h k l) / \overline{f^{2}}(h k l)}{\sum_{i=1}^{t} Z_{i}^{2}-U_{n_{1} o} U_{n_{2} o} U_{n_{3} o} \cdot F^{2}(000)}, \text { (3) }
$$

where $\Sigma^{\prime}$ denotes the sum $\sum_{h=-\infty}^{\infty} \sum_{k=-\infty}^{\infty} \sum_{l=-\infty}^{\infty} \theta$.

cluding the term of $h=k=l=0$. $U_{n h}$ are known functions of $n$ and $h$, the numerical values of which are negligibly small for $h>n / 2 ; U_{n h}=U_{n} \vec{h}$ when $\bar{h}=-h$. Thus, if the maximum $h, k, l$ of our available set of $I^{\prime}(h k l)$ are $h_{M}, k_{M}, l_{M}$, we may choose $n_{1} \doteq 2 h_{M}$, $n_{2} \doteq 2 k_{M}, n_{3} \doteq 2 l_{M}$. The values of $U_{n h}$ for some values of $n$ and $h$ are given below :

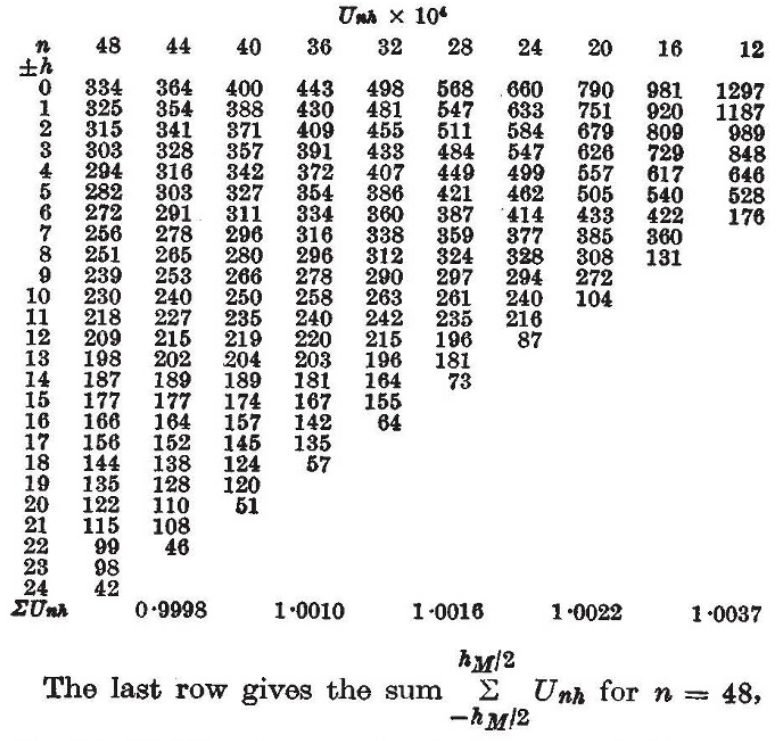

40, 32, 24, 16. Apparently all the sums differ from the theoretical limit $\sum_{h=-\infty}^{\infty} U_{n h}=1$ only by fractions of 1 per cent, indicating the rapidity of convergence of the infinite series.

The accuracy of $I^{\prime}(000)$ deduced from (3) depends essentially upon the following four factors:

(1) The accuracy of the observed $I^{\prime}(h k l)$.

(2) The order of approximation of the equation

$$
f_{i}(h k l)=Z_{i} \cdot \bar{f}(h k l) \text {, }
$$

which forms the basis of equation (2). The error introduced by the failure of equation (4) is usually not very serious.

(3) The proper correction of the temperature effect in $f_{i}(h k l)$. If we actually do a suitable experiment to correct this effect, the error introduced is, of course, limited. Otherwise we have to correct the effect with reference to similar crystals of known structures, and 
the error resulting in $I^{\prime}(000)$ may be very large. This uncertain correction of the temperature effect constitutes the chief source of errors in the value deduced for $I^{\prime}(000)$.

(4) The correct choice of $n_{1}, n_{2}, n_{3}$. The strict condition is

$$
d_{m} \geqslant \frac{1 \cdot 2 a}{n_{1}}, \frac{1 \cdot 2 b}{n_{2}}, \frac{1 \cdot 2 c}{n_{8}},
$$

where $d_{m}$ denotes the shortest interatomic distance between any two atoms in contact in the crystal. Because this condition is rather too strict to be satisfied easily. let us introduce a much more approximate condition,

$$
\begin{aligned}
d_{a} \geqslant & 1 \cdot 2\left\{\left(\frac{a}{n_{1}}\right)^{2}+\left(\frac{b}{n_{c}}\right)^{2}+\frac{2 a b}{n_{1} n_{2}}|\cos \gamma|\right\}^{1 / 2}, \\
& 1 \cdot 2\left\{\left(\frac{b}{n_{2}}\right)^{2}+\left(\frac{c}{n_{3}}\right)^{2}+\frac{2 b c}{n_{2} n_{8}}|\cos \alpha|\right\}^{1 / 2}, \\
& 1 \cdot 2\left\{\left(\frac{a}{n_{1}}\right)^{2}+\left(\frac{c}{n_{3}}\right)^{2}+\frac{2 a c}{n_{1} n_{3}}|\cos \beta|\right\}^{1 / 2},
\end{aligned}
$$

where $d_{a}$ denotes the average interatomic distance between any two contact atoms in the crystal : $d_{a}$ can be roughly estimated from the nature of the atoms contained. In case the structure contains a number of very heavy small atoms, their effect on $I^{\prime}(000)$ can be estimated.

When all of the foregoing four points have been provided for, it is believed that the error in $I^{\prime}(000)$ would fall in the range of $5-20$ per cent in the usual cases.

To illustrate the use of (3), let us first take a crystal consisting of only one atom at the origin in the unit cell, the number of electrons in the shell of the atom being $Z$ and the atomic structure factor, $f(h k l)$. Then $F(h k l)=f(h k l)=Z \bar{f}(h k l) . F^{2}(000)=Z^{2}$. If we assume $I^{\prime}(h k l)=F^{2}(h k l)$, we have $I^{\prime}(h k l)$ j $\bar{f}^{2}(h k l)=Z^{2}$. Substituting these values in (3) we obtain

$$
\begin{aligned}
& I^{\prime}(000)=
\end{aligned}
$$

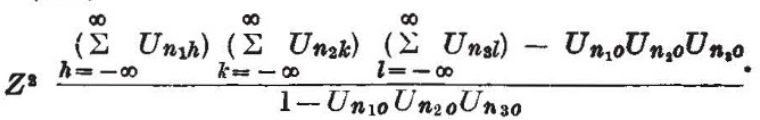

Using $U_{n h}$ given in the table, with $n_{1}=48, n_{2}=32$, $n_{3}=16$, we obtain $I^{\prime}(000)=1 \cdot 0051 Z^{2}$, which differs from the assumed $I^{\prime}(000)=F^{2}(000)=Z^{2}$ only by 0.51 per cent. The choice of $U_{n h}$ of any other $n_{1}$, $n_{2}, n_{3}$ will give similar results.

Let us next illustrate the method by studying a real erystal, say, paradinitrobenzene, the structure of which was determined carefully by James, King and Horrocks ${ }^{4}$, who made the determination on the basis of intensities of reflexions $h k 0, h 0 l$ and $0 k l$. Strictly speaking, we should determine $I^{\prime}(000)$ from equation (3), based on the relative intensities of the whole set of reflexions $h k l$. But in the case of the present structure, as we know already from James, King and Horrocks' paper that there is no overlapping of atoms directly upon each other in the projection of the structure in direction $b$ to the $a c$ plane (see ref. 4, Fig. 3), a study of the relative intensities of reflexions $h 0 l$ is sufficient for our purpose of illustra. tion. Then let us take their observed $F^{2}(h 0 l)$ directly as our $I^{\prime}(h 0 l)$ and take Robertson's $f$-values ${ }^{8}$ directly as our $\bar{f}(h 0 l)$. Since no further temperature correction has been made, the values of $\bar{f}(h 0 l)$ so obtained are under-corrected for the temperature effect as revealed from my studies of the same structure. But we may deliberately use such rough values of $\bar{f}(h k l)$ in order to se日 if $I^{\prime}(000)$ so deduced still proves reliable. The structure has

$$
F^{2}(000)=164^{2}, \sum_{i=1}^{t} Z_{i}^{2}=1140
$$

when the contribution of the hydrogen atoms is neglected. $h_{M}=13$ such as in $h 0 l=1301$, and $l_{M}=7$ such as in $h 0 l=107$. Accordingly, we take $n_{1}=24, n_{3}=12$. The two-dimensional equation for $I^{\prime}(000)$ is given by

$$
I^{\prime}(000)=F^{2}(000) \frac{\sum^{\prime} U_{n_{1} h} U_{n_{3}} I^{\prime}(h 0 l) / \overline{f^{2}}\left(h^{\wedge} l\right)}{\sum_{i=1}^{t} Z_{i}{ }^{2}-U_{n_{1} o} U_{n_{3} o} F^{2}(000)},
$$

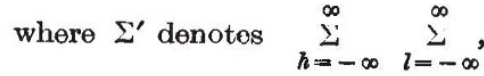

excluding the term with $h=l=0$. Substituting in $(3 a)$ the appropriate $U_{n h}$ from the table and the other quantities mentioned above, we obtain $I^{\prime}(000)=$ $0 \cdot 85 F^{2}(000)$, differing from the assumed $I^{\prime}(000)=$ $F^{2}(000)$ by 15 per cent ; an acceptable error obtained by deliberately choosing rather rough values of $\bar{f}(h k l)$.

Details of this work, including the method of correcting for the effect of small heavy atoms, will be presented elsewhere.

${ }^{1}$ Booth, A. D., Nature, 158. 380 (1946). Boyes-Watson, J., Davidson, E., and Perutz, M. F., Proc. Roy Soc, A 191, 83 (1947). Harken, D.' and Kasper, J.'S., Reports of ' 1947 summer meeting ASEXRD, 13. Buerger, M. J., Phys. Rev., 73, 927 (1948).

${ }^{2}$ Booth, A. D., Kasper, J. S., Reports of 1947 summer meeting of ASEX RD, 14.

Yü, S. H., Nature, 1E0, 151 (1942).

- James, R. W., King, G., and Horrocks, H., Proc. Roy. Soc., A, 158 878 (1935).

- Robertson, J. M., Proc. Roy. Soc., A, 150, 109 (1935).

\section{$30 \%$}

\section{FRACTURE OF SOLIDS}

A CONFERENCE on "The Fracture of Solids" was held 'by the British Rheologists' Club on October 29. P. f. N. F. Mott, recently elected president $\mathrm{W}$ the Club, opened the conference by pointipg oth that, quite apart from its practical in poprince, fracture was the most interesting prpperty of solids to the theoreticians because it is the least understood property, no progress having been made beyond the Griffith crack-theory of 1924. It is not known how cracks exist nor what causes them; experimental work has made clear what happens during fracture, but not how it occurs.

Dr. C. F. Tipper then presented a paper describing some beautiful experimental work on the subject of fracture in mild steel. She divided fracture into three types: the first corresponds to a parting along a shear plane; the second along two intersecting planes, giving a wedge-shaped fracture; and the third along a plane quite different from the shear plane, often at right angles to the principal tensile stress. Iron and mild steel show all three types of fracture, and it is possible to have all in the same test-piece, even in adjacent crystals. The amount of energy absorbed in fracture depends on how much of each type goes to make up the total fracture. The third type, which is generally brittle, can always be 